\title{
Searches for vector-like quarks at CMS
}

\author{
Justin Pilot, on behalf of the CMS Collaboration* \\ University of California, Davis \\ E-mail: jrpiloteucdavis.edu
}

This contribution details results of recently completed searches for massive vector-like top and bottom quark partners using proton-proton collision data collected with the CMS detector at the CERN LHC at a center-of-mass energy of $13 \mathrm{TeV}$. Single and pair production of vector-like quarks are studied, with decays into a variety of final states, containing top and bottom quarks, electroweak gauge and Higgs bosons. Analyses utilize several categories of reconstructed objects, from multi-leptonic to fully hadronic final states. Improved exclusion limits on both the vectorlike quark mass and cross sections are obtained, for combinations of the possible vector-like quark branching ratios.

The 39th International Conference on High Energy Physics (ICHEP2018)

4-11 July, 2018

Seoul, Korea

${ }^{*}$ Speaker. 


\section{Introduction}

Vector-like quarks (VLQs) are a feasible solution to the hierarchy problem, and arise in many extensions to the SM. These particles are named vector-like because their left- and right-handed components transform the same way under the $S U(2)$ symmetry group. The VLQs presented here are denoted $T$ and $B$. The $T(B)$ quark has three dominant decay modes: $t H, t Z$, and $b W(b H, b Z$, and $t W$ ). A VLQ singlet scenario is studied, with branching ratios to $t W / b W$ bosons being $50 \%$, and branching ratios to $b Z / t Z$ and $b H / t H$ being $25 \%$ each. A doublet scenario is also considered, with no decay to $t W / b W$, and the other branching ratios equivalent.

There are two production mechanisms for VLQs at the Large Hadron Collider (LHC). The pair production mode is through the strong interaction, and is essentially model independent, while the single production mode requires associated forward jets, and depends strongly on the couplings to SM quarks. Vector-like quarks are expected to be produced at the LHC with high rates, with the expected production cross sections ranging from 0.1 to $1000 \mathrm{fb}$ for VLQs with masses from 1600 to $600 \mathrm{GeV}$ [2]. Above $600 \mathrm{GeV}$, single VLQ production begins to dominate relative to the pair production mode, as they have received a greater enhancement from the change in LHC center-of-mass energy from 8 to $13 \mathrm{TeV}$.

This presentation will summarize the recent VLQ search results from the CMS experiment [1].

\section{Search for VLQs using leptons}

A recent search for VLQ pair production $T T$ or $B B$ has been completed utilizing leptons in the final state [3]. The combination of different lepton counts in the final state with jet substructure techniques to identify hadronic decays of $W$ or Higgs bosons enhances the sensitivity to the VLQ signal.

The search encompasses several unique categories. In the single lepton category, events are divided based on the number of identified $W, H$, and $b$ tagged objects. Depending on the signal region, the quantity $\min [M(\ell, b)]$ is computed to separate the top quark pair background from the VLQ signal by finding the lepton and $\mathrm{b}$ quark combination that gives the lowest reconstructed mass. This quantity will be higher for VLQ events

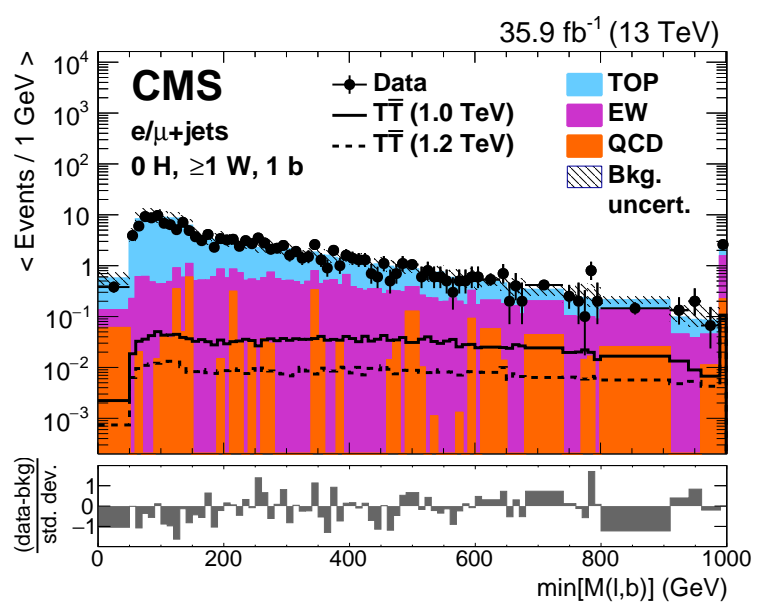

Figure 1: Distribution of $\min [M(\ell, b)]$, used to discriminate signal from background in the search for VLQ pairs using leptons. [3] compared to SM backgrounds, as seen in Fig 1. In other categories, the sum of lepton and jet energies, $S_{T}$, is used to discriminate the signal.

In the same-sign dilepton and trilepton categories of this analysis, backgrounds from charge misidentification and non-prompt leptons are taken into account, with events containing non- 
prompt leptons being the leading contribution in the trilepton signal regions. For the same-sign dilepton signal regions, a simple counting experiment is performed, while for the trilepton signal regions, the $S_{T}$ distribution is used to discriminate signal.

A scan over branching ratios is performed, with VLQ mass exclusion limits ranging from 900 to $1300 \mathrm{GeV}$. For the singlet scenario, $T$ quarks with masses below $1.2 \mathrm{TeV}$ are excluded $(1.16 \mathrm{TeV}$ expected), while $B$ quarks with masses below $1.17 \mathrm{TeV}$ are excluded (1.13 $\mathrm{TeV}$ expected). These results represent significant improvements upon Run 1 results.

A complementary analysis [5] also uses leptons to search for singly-produced $T$ quarks, using the $t Z$ decay mode, excluding a VLQ $T$ quark with mass below $1.2 \mathrm{TeV}$ in the singlet scenario.

\section{Targeting $B \rightarrow b H$ with Higgs tagging}

Another recent analysis utilizes the single VLQ production mode to search for single $B$ quarks produced in association with a forward jet [4]. Requiring this forward jet enhances the signal purity, as SM events tend to have fewer forward jets compared to the single VLQ signature, as seen in Figure 2. In addition, the analysis targets the specific $b H$ decay mode of the $B$ quark. Using subjet $\mathrm{b}$ tagging as well as jet mass observables, the Higgs signature can be efficiently identified while rejecting SM backgrounds.

The analysis divides events based on the number of forward jets, and uses a data-driven method to estimate the dominant QCD multijet background. That background is estimated using control regions formed by inverting requirements on the Higgs candidate jet mass and the number of subjet $\mathrm{b}$ tags found. Finally, the invariant mass of the $\mathrm{b}$ and Higgs candidates is computed to discriminate the VLQ signature, where a peak is expected relative to the SM background.

This search for $b H$ yields cross section limits in the range of 1.28 to $0.07 \mathrm{pb}$, for $B$ masses between 0.7 and $1.8 \mathrm{TeV}$. Currently, no mass exclusion is obtained in the search range, however, this is the first analysis to assume a non-negligible VLQ width and include its effects in the signal model.

\section{Search in $t W$ channel with jet substructure}

The final analysis to be discussed is again a search for $B$ quarks in the single production mode, but targeting the $t W$ decay mode [6]. At least one electron or muon is required in this analysis. Jet substructure techniques are used to identify boosted hadronic top quark or $\mathrm{W}$ boson candidates. Events are then divided based on the number of these objects identified in the final

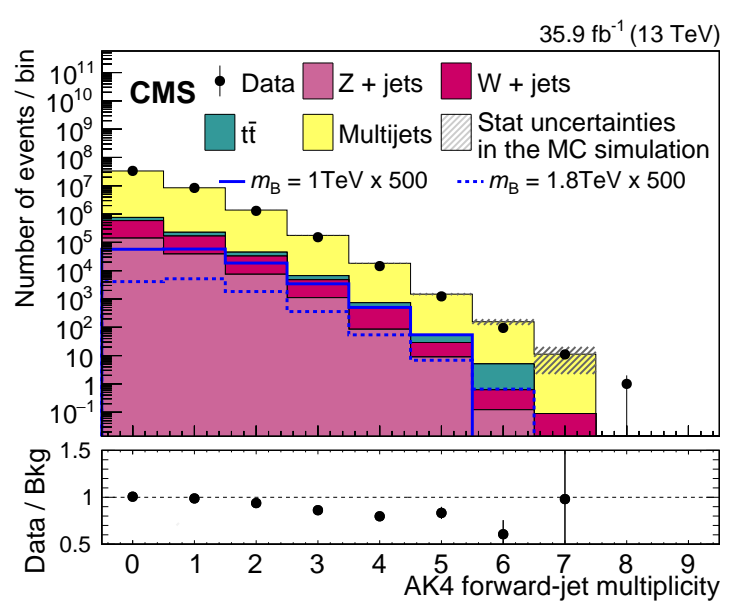

Figure 2: The distribution of the number of forward jets in selected events, with the VLQ signal peaking at larger values than the background. [4] 
state. The mass of the $B$ quark is recon-

structed by combining the top quark and leptonic $W$ candidates. A $\chi^{2}$ method is used to assign objects to the top quark and/or $W$ boson candidate in other signal regions. Minimizing this $\chi^{2}$ quantity, including terms for the top quark and $W$ boson masses, as well as the angular separation of the two, provides the best resolution and signal discrimination.

The backgrounds in all signal regions are estimated from data by inverting the requirement of at least one forward jet in the event. Shapes from this control region are then extrapolated to the signal region. One of the signal regions, requiring a boosted top jet candidate, is shown in Figure 3. Limits are set on VLQ $B$ production, excluding cross sections above $0.3 \mathrm{pb}$ for a $B$ quark mass of $700 \mathrm{GeV}$, and cross sections above 0.03 pb for a $B$ quark mass of 1.8 $\mathrm{TeV}$.

\section{Summary}

This document details some of the recent results in the search for vector-like quarks by the CMS collaboration. Searches targeting the single production mode are becoming more powerful in Run 2 due to the greater increase in cross section relative to the pair production mode. The full set of results of CMS searches for VLQs can be found at Ref. [7].

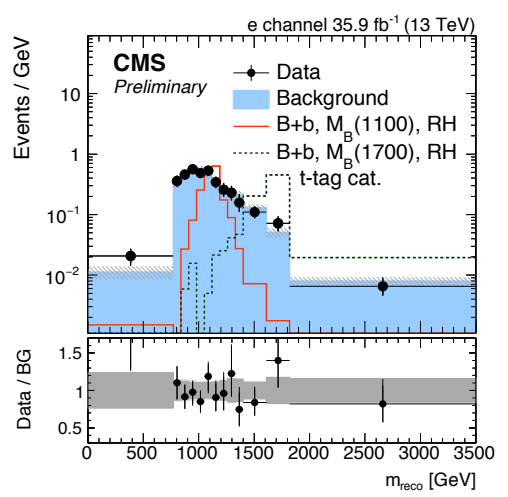

Figure 3: Data-driven background for the signal region containing a boosted top jet candidate. [6]

\section{References}

[1] CMS Collaboration, "The CMS experiment at the CERN LHC", JINST 3 (2008) S08004, https://doi.org/10.1088/1748-0221/3/08/S08004

[2] J. A. Aguilar-Saavedra, R. Benbrik, S. Heinemeyer, and M. Pérez-Victoria, "Handbook of vectorlike quarks: Mixing and single production", Phys.Rev.D 88 (2013) 094010, https://doi.org/10.1103/PhysRevD.88.094010

[3] CMS Collaboration, "Search for vector-like T and B quark pairs in final states with leptons at $\sqrt{s}=13$ TeV", JHEP 08 (2018) 177, http://dx.doi.org/10.1007/JHEP08(2018)177

[4] CMS Collaboration, "Search for single production of vector-like quarks decaying to a b quark and a Higgs boson", JHEP 06 (2018) 031, http://dx.doi.org/10.1007/JHEP06(2018)031

[5] CMS Collaboration, "Search for single production of a vector-like $\mathrm{T}$ quark decaying to a $\mathrm{Z}$ boson and a top quark in proton-proton collisions at $\sqrt{s}=13 \mathrm{TeV}$ ", Phys.Lett.B 781 (2018) 574, http://dx.doi.org/10.1016/j.physletb.2018.04.036

[6] CMS Collaboration, "Search for a vector-like quark decaying to a top quark and a W boson", CMS-PAS-B2G-17-018 (2018), http://cds.cern.ch/record/2628654

[7] CMS Collaboration, "Beyond-two-generations Public Physics Results", https://twiki.cern.ch/twiki/bin/view/CMSPublic/PhysicsResultsB2G 